\title{
Nonholonomic and Stratified Robotic Manipulation Supplemented with Fuzzy Control: Theory and Experiment
}

\author{
Neil Petroff and Bill Goodwine
}

\begin{abstract}
This paper presents initial results in the novel integration of nonholonomic and stratified motion planning, fuzzy control and tactile sensing to construct a robotic manipulation system that is designed to be both dexterous and robust. It is dexterous in that it is fully nonlinear, can explicitly incorporate discontinuities in the motion planning i.e., finger gaiting, and allows rolling finger contacts. The sensing and fuzzy controller are intended to provide robustness that is necessary for realworld manipulation tasks that are characterized by modeling errors and are subjected to unmodeled external disturbances. The method is demonstrated experimentally using a set of four robots with end-effectors equipped with force sensors to provide feedback to the fuzzy supervisory control system.
\end{abstract}

\section{INTRODUCTION}

The ultimate goal of this work is to effectively combine aspects of nonlinear stratified motion planning with fuzzy logic and tactile sensing to provide operational flexibility and robustness to a set of cooperating robot manipulators acting as fingers to dexterously manipulate objects. While nonlinear nonholonomic motion planning methods are very general, the cost of such breadth is reduced robustness because a nearly exactly parametrized model is necessary. Furthermore, most manipulation systems are modeled as purely kinematic, which, if force closure is maintained, eliminates the effect of gravity in the model. However, no system is truly kinematic, and in this paper, the main external disturbance affecting the manipulation is due to gravity.

The addition of a fuzzy logic supervisory controller interfaced with a tactile sensing is intended to provide a layer of robustness with respect to manipulation requirements. Fig. 1 shows the experimental platform that will be fully explained subsequently. The main contributions of this paper are preliminary results from a investigation of the combination of these methods as well as an experimental demonstration. The most closely related work is from HKUST [10], [12]-[14], etc., as well as [8]. The distinguishing feature of the work in this paper is using fuzzy control as a supplement to enhance the effectiveness of the manipulation system. Fuzzy logic has been used previously in manipulation systems [2], but not specifically coupled with the methods used herein and is not focused on finger repositioning.

The authors gratefully acknowledge the support of the TARDEC through the SimBRS program and a the National Science Foundation through IIS9910602-SGER for the experimental platform.

Neil Petroff is with I/N Kote I/N Tek, New Carlisle, IN Neil.Petroffearcelormittal.com

Bill Goodwine is with the Department of Aerospace \& Mechanical Engineering, University of Notre Dame, Notre Dame, IN 46556 USA billecontrols.ame.nd.edu

UNCLASSIFIED: Dist A. Approved for public release.

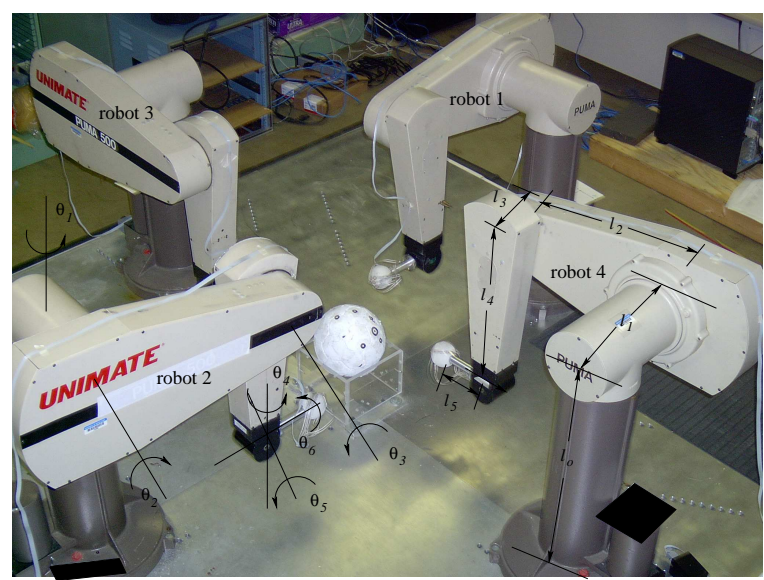

Fig. 1. Robotic manipulation test bed.

The abilities humans possess in using tactile sensing in dealing with unstructured environments provides motivation for our approach. A block diagram of the control system we use is illustrated in Fig. 2. Early work in motion planning was done with continuous, holonomic systems [7], [16], [24]. Such approaches would be applicable to static grasps; however, for present purposes, it is desired to accommodate fingers rolling on the surface of the manipulated object and to also allow for discontinuous contact. Much of the previous work of one of the authors was based on extending the the nonholonomic motion planning method from [9] (and related work [1], [7], [18]) to the stratified case to apply it to robotic manipulation and locomotion [3]-[5], [27].

Many researchers use tactile sensing and types of position/force control which uses information about contact force. For example, [19], [34] use force feedback, but on the wrist, away from the contact interface, [29] assumes contact forces can be measured and [25] provides a method to calculate force at the end-effector. This paper presents a new approach which combines a fuzzy supervisor using configuration and contact force feedback with the open loop motion planning algorithm designed to help maintain force closure and to allow periodic trajectory recalculations.

Fuzzy logic has a long history in control, specifically applied to robotics. In typical applications, a supervisor monitors system performance and effects switching between various plant models [11], for example, in [23] for trajectory planning of a hovercraft and to stabilize a small-scale helicopter after rotor failure [15]. Spong [22] has suggested that human ambulation is too complicated to achieve with a single controller and robust grasping likely offers similar 


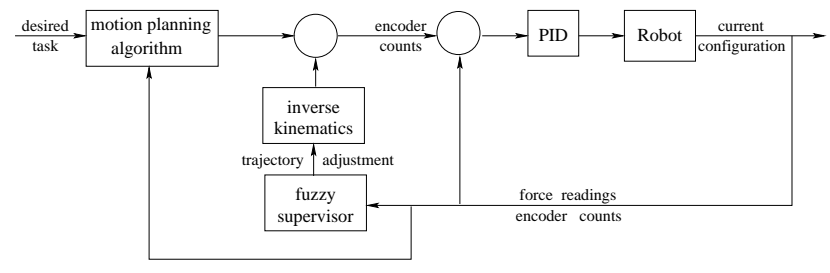

Fig. 2. Control architecture for the manipulation task.

complexity. Some suggest fuzzy logic is an excellent representation for biological systems due to their shared empirical properties, [30]. In addition, evidence suggests that the brain uses a set of quantitative rules to determine activation levels in muscle synergy [28]. In our implementation in Fig. 2, the fuzzy supervisor block uses a fuzzy inference system to adjust the trajectories of the fingertips at certain key junctures during the manipulation task.

\section{CONTROL FrAMEWORK}

This section provides a brief overview of each of the topics that are combined in this research.

\section{A. Nonlinear and Stratified Motion Planning}

In the usual application of nonholonomic motion planning to grasping, a trajectory is manifested in moving a finger from one position to another on an object while maintaining contact. In the case of stratified manipulation [3], exploiting the discontinuities in the system that arise from intermittent contact are also considered. In either case, the motion planning method is a means to determine control inputs for

$$
\dot{x}=g_{1}(x) u_{1}+g_{2}(x) u_{2}+\cdots+g_{m}(x) u_{m},
$$

where the $g_{i}(x)$ are vector fields and $u_{i}$ are control inputs, that will steer the system from a starting point to a desired final point. As is always the case in grasping, since the object itself is not actuated, the system is underactuated because the number of inputs is less than the dimension of the configuration space. The adopted, [9], method works exactly for nilpotent systems and locally for nonnilpotent systems (see [17]), and approximately otherwise. The general approach to solving Eq. 1 for the inputs to generate a desired trajectory is:

1) determine the Philip Hall basis for the system which eliminates the linear dependence of some vector fields due to the Jacobi identity;

2) construct the extended system

$$
\begin{aligned}
\dot{x}=g_{1}(x) u_{1}+ & g_{2}(x) u_{2}+\cdots+g_{m}(x) u_{m} \\
& +g_{m+1}(x) u_{m+1}+\cdots+g_{s}(x) u_{s},
\end{aligned}
$$

where $g_{1}, \ldots, g_{m}$ are from Eq. 1 and $g_{m+1}, \ldots, g_{s}$ are Lie brackets such that the distribution $\bar{\Delta}=$ $\operatorname{span}\left\{g_{1}, \ldots, g_{s}\right\}$ is full rank;

3) if stratified planning will be used, repeat the previous step for each combination of fingers that may be in contact with the object, i.e., for each stratum;

UNCLASSIFIED

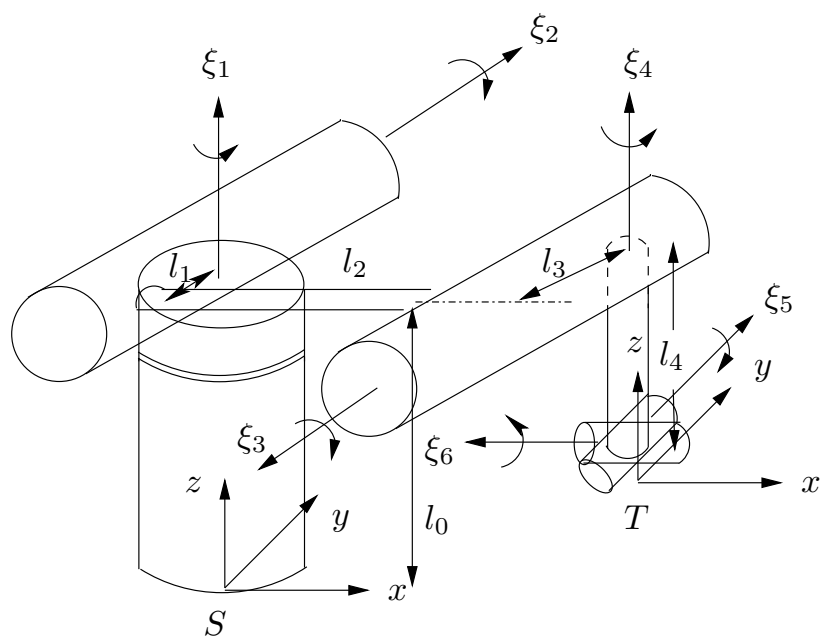

Fig. 3. Zero configuration of PUMA 560 showing frame orientations and twists.

4) determine the inputs for the extended system, where the first $m$ of these inputs corresponds to the original system and the remaining $s-m$ inputs are "fictitious" inputs that correspond to Lie bracket motions; and,

5) convert the fictitious inputs to those produced through Lie bracket motions using a sequence of piece-wise constant inputs.

The extension to the stratified case is in [5], [6].

\section{B. Kinematics of Robotic Manipulation and Grasping}

We will make use of the usual product of exponentials formulation as developed in [17] where the configuration of a tool frame $T$ relative to a base frame $S$ is given by

$$
g_{s t}(\theta)=e^{\hat{\xi}_{1} \theta_{1}} e^{\hat{\xi}_{2} \theta_{2}} \cdots e^{\hat{\xi}_{n} \theta_{n}} g_{s t}(0),
$$

where $n$ is the number of joints on the robot. Our experimental platform uses PUMA 560 manipulators, the basic kinematics of which are schematically illustrated in Fig. 3.

We also make use of the grasp map, as developed in [17]. Generally, a wrench $W_{i}$ that a finger can apply to an object at contact $i$ has the form $W_{i}=B_{i} f_{i}$, where $B_{i}$ is the wrench basis indicating directions in which wrenches can be applied based on the finger model and $f_{i}$ is the vector of magnitudes of the applied force components that must be consistent with the limits allowed by friction. In this work, because of the nature of the fingertips (described subsequently), a soft finger contact model is used. Therefore,

$$
W_{i}=\left[\begin{array}{llll}
1 & 0 & 0 & 0 \\
0 & 1 & 0 & 0 \\
0 & 0 & 1 & 0 \\
0 & 0 & 0 & 0 \\
0 & 0 & 0 & 0 \\
0 & 0 & 0 & 1
\end{array}\right]\left[\begin{array}{c}
f_{x} \\
f_{y} \\
f_{z} \\
\tau
\end{array}\right] .
$$

Ultimately we need an expression for the constraint between the finger joint velocities and the object velocity. We use the usual notation for grasping problems: 


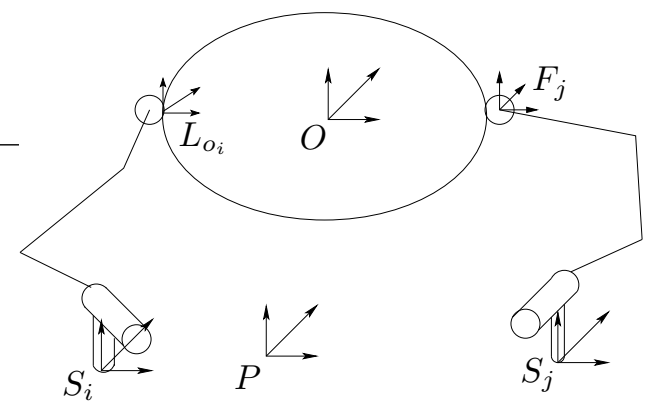

Fig. 4. Frames for manipulation system.

- $c_{o}\left(u_{o}, v_{o}\right)$ and $c_{f}\left(u_{f}, v_{f}\right)$ are the orthogonal surface parameterizations for the object and fingers, respectively;

- $O$ is a reference frame affixed to the object, $F_{i}$ is a reference frame affixed to finger $i$ and $P$ is a common palm frame; and,

- Gauss frames are defined at each point on the surface of the fingers and object and also two additional local frames are defined on the object $L_{o}$, and finger, $L_{f}$ for all time the two objects are in contact which are fixed with respect to $O$ and $F_{i}$, respectively and coincide with the Gauss frames at the point of contact.

These are schematically illustrated in Fig. 4.

A fundamental grasp constraint can be developed relating directions in which relative motion between a finger and an object is precluded. In general for the contact between the $i$ th finger and the object, this is given by

$$
B^{T} V_{l_{o} l_{f}}^{b}=0 \text {, }
$$

where $V_{l_{o} l_{f}}^{b}$ is the body velocity of a frame $L_{o}$ attached to the object at the contact point relative to the $L_{f}$ frame at the same point but attached to the finger, and $B$ is the wrench basis for the model of finger $i$. Hence, if we denote $V_{l_{o} l_{f}}^{b}=$ $\left[\begin{array}{llllll}v_{x} & v_{y} & v_{z} & \omega_{x} & \omega_{y} & \omega_{z}\end{array}\right]^{T}$ then Eq. 3 gives

$$
V_{l_{o} l_{f}}^{b}=\left[\begin{array}{llllll}
0 & 0 & 0 & \omega_{x} & \omega_{y} & 0
\end{array}\right]^{T}
$$

which represents the fact that, for a soft finger contact, the only relative velocity between the finger and object that is allowed is relative rolling between the surfaces.

Based on [16], the contact point between each finger rolling on the surface of an object evolves according to

$$
\begin{aligned}
\dot{\alpha}_{f} & =M_{f}^{-1}\left(K_{f}+\tilde{K}_{o}\right)^{-1}\left(\left[\begin{array}{c}
-\omega_{y} \\
\omega_{x}
\end{array}\right]-\tilde{K}_{o}\left[\begin{array}{l}
v_{x} \\
v_{y}
\end{array}\right]\right) \\
\dot{\alpha}_{o} & =M_{o}^{-1} R_{\psi}\left(K_{f}+\tilde{K}_{o}\right)^{-1}\left(\left[\begin{array}{c}
-\omega_{y} \\
\omega_{x}
\end{array}\right]+K_{f}\left[\begin{array}{l}
v_{x} \\
v_{y}
\end{array}\right]\right) \\
\dot{\psi} & =\omega_{z}+T_{f} M_{f} \dot{\alpha}_{f}+T_{o} M_{o} \dot{\alpha}_{o} \\
v_{z} & =0
\end{aligned}
$$

where $\alpha$ represents the local point $(u, v)$ and the subscripts $f$ and $o$ are for the finger and object, respectively. The geometric parameters, $M, K$, and $T$, are the metric tensor, curvature tensor and torsion tensors, respectively. For a soft

UNCLASSIFIED finger contact, the sliding velocity components, $v_{x}$ and $v_{y}$ are zero. However, as will be be outlined subsequently, these sliding velocities will correspond to Lie bracket directions, and hence are retained in the above equations. The modified curvature tensor, $\tilde{K}_{o}$, is given by $\tilde{K}_{o}=R_{\psi} K_{o} R_{\psi}$ with

$$
R_{\psi}=\left[\begin{array}{cc}
\cos \psi & -\sin \psi \\
-\sin \psi & -\cos \psi
\end{array}\right],
$$

where $\psi$ is the contact angle between the $x$-axes of Gauss frames affixed to the object and finger at the point of contact.

\section{Rolling-Manipulation Constraint Equation}

The implementation utilized requires that the equations of motion be formulated in a specific manner to allow for appropriate construction of the extended system. The constraints for the moving contact-location grasp, i.e., rolling, can be written by traversing frame origins from the local frame on the object at the point of contact $L_{o}$, through the kinematics of the system to the finger at the point of contact $L_{f}$. The configuration of the local finger frame relative to the local object frame for a single finger can be written as $g_{l_{o} l_{f}}=g_{l_{o} o} g_{o p} g_{p s} g_{s f} g_{f l_{f}}$ and the body velocity of the relative frames is then $\hat{V}_{l_{o} l_{f}}^{b}=g_{l_{o} l_{f}}^{-1} \dot{g}_{l_{o} l_{f}}$, where

$$
g_{l_{o} l_{f}}^{-1}=g_{f l_{f}}^{-1} g_{s f}^{-1} g_{p s}^{-1} g_{o p}^{-1} g_{l_{o} o}^{-1}
$$

and

$$
\begin{aligned}
\dot{g}_{l_{o} l_{f}}= & g_{l_{o} o} g_{o p} g_{p s} g_{s f} \dot{g}_{f l_{f}}+g_{l_{o} o} g_{o p} g_{p s} \dot{g}_{s f} g_{f l_{f}} \\
& +g_{l_{o} o} g_{o p} \dot{g}_{p s} g_{s f} g_{f l_{f}}+g_{l_{o} o} \dot{g}_{o p} g_{p s} g_{s f} g_{f l_{f}} \\
& +\dot{g}_{l_{o} o} g_{o p} g_{p s} g_{s f} g_{f l_{f}} .
\end{aligned}
$$

Hence

$$
\begin{aligned}
\hat{V}_{a b}^{b}= & g_{l_{o} l_{f}}^{-1} \dot{g}_{l_{o} l_{f}}=g_{f l_{f}}^{-1} \dot{g}_{f l_{f}}+g_{f l_{f}}^{-1}\left(g_{s f}^{-1} \dot{g}_{s f}\right) g_{f l_{f}} \\
& +g_{f l_{f}}^{-1} g_{s f}^{-1}\left(g_{p s}^{-1} \dot{g}_{p s}\right) g_{s f} g_{f l_{f}}+\cdots .
\end{aligned}
$$

Switching from matrix to vector form then gives

$$
\begin{aligned}
V_{l_{o} l_{f}}^{b}=V_{f l_{f}}^{b}+ & \operatorname{Ad}_{g_{f l_{f}}}^{-1} V_{s f}^{b}+\operatorname{Ad}_{g_{f l} l_{f}}^{-1} \operatorname{Ad}_{g_{s f}}^{-1} V_{p s}^{b} \\
& +\operatorname{Ad}_{g_{f l_{f}}}^{-1} \operatorname{Ad}_{g_{s f}}^{-1} \operatorname{Ad}_{g_{p s}}^{-1} V_{o p}^{b} \\
& +\operatorname{Ad}_{g_{f l_{f}}}^{-1} \operatorname{Ad}_{g_{s f}}^{-1} \operatorname{Ad}_{g_{p s}}^{-1} \operatorname{Ad}_{g_{o p}}^{-1} V_{l_{o} o}^{b} .
\end{aligned}
$$

Noting that

- $V_{f l_{f}}^{b}=V_{p s}^{b}=V_{l_{o} o}^{b}=0$;

- $V_{s f}^{b}=\operatorname{Ad}_{g_{s f}}^{-1} J_{s f}^{s} \dot{\theta}_{f}$, where $J_{s f}^{s}$ is the spatial Jacobian of the finger frame with respect to the station frame;

- $\operatorname{Ad}_{g_{f l_{f}}}^{-1} \operatorname{Ad}_{g_{s f}}^{-1} \operatorname{Ad}_{g_{p s}}^{-1}=\operatorname{Ad}_{g_{p l} l_{f}}^{-1}$;

- $\operatorname{Ad}_{g_{f l_{f}}}^{-1} \operatorname{Ad}_{g_{s f}}^{-1}=\operatorname{Ad}_{g_{s l_{f}}}^{-1}$; and,

- $V_{o p}^{b}=-V_{p o}^{s}$,

the velocity can be written as

$$
V_{l_{o} l_{f}}^{b}=\operatorname{Ad}_{g_{s l_{f}}}^{-1} J_{s f}^{s} \dot{\theta}_{f}-\operatorname{Ad}_{g_{p l_{f}}}^{-1} V_{p o}^{s} .
$$

Substituting Eq. 4 into Eq. 7 and solving for $\dot{\theta}$ gives the joint velocities for each finger

$$
\dot{\theta}_{f_{i}}=\left(J_{s_{i} f_{i}}^{s}\right)^{-1}\left[\operatorname{Ad}_{g_{p s_{i}}}^{-1} V_{p o}^{s}+\operatorname{Ad}_{g_{s_{i} l_{i}}} \xi_{i}\right]
$$


where

$$
\xi_{i}=\left[\begin{array}{llllll}
0 & 0 & 0 & \omega_{x_{i}} & \omega_{y_{i}} & 0
\end{array}\right]^{T}
$$

Eq. 8 is the rolling-contact constraint equation, and there will be one for each point of contact. It describes manipulator joint velocities necessary to achieve some time-dependent, rigid-body velocity of the object while maintaining rolling contact. Note that Eq. 8 is not general in that the number of actuated joints is exactly what is necessary to invert the adjoint transformation and directly solve for the joint velocities. If the actuator has additional joints, then a pseudoinverse could be used. If it has fewer joints, then methods from stratified manipulation need to be used.

\section{Fuzzy Logic and Control}

The tool used for feedback correction for the manipulation algorithm is fuzzy logic and control. The mainstream engineering introduction of fuzzy logic is generally from the work of Zadeh [31]-[33]. Fuzzy control is particularly appealing as a supplement to nonlinear motion planning in grasping because it is not model-based so the difficulties of determining nearly exact models in nonlinear control is alleviated and it provides a relatively straight-forward means to translate heuristics into effective control algorithms. The basic component of fuzzy logic is the membership function. The traditional notion of a set requires that something is either an element of the set or it is not. In contrast, a fuzzy set allows partial degrees of membership, and the membership functions used in this work are illustrated in Fig. 9.

Much of the structure of classical logic can be generalized based upon the notion of fuzzy sets. What this allows, then, is for a linguistic description of a control algorithm to be translated into a system that is easily implementable. It is straightforward to combine multiple rules with multiple inputs to achieve relatively complicated control algorithms based upon effective control approaches that are based upon heuristics. An overview can be found in [21], and the approach is fleshed out more in Section III which describes our implementation, which is based to some degree on [20].

\section{IMPLEMENTATION}

The experimental platform consists of four, six-degree-offreedom Unimate PUMA 560 robots illustrated in Fig. 1. The robots are fixed on a 94" by 94" raised platform equidistant from the platform's center. Pliable balls which are 2.75" in diameter are used for fingertips. Six force sensors, purchased from Tekscan, and sold under the product name of FlexiForce ${ }^{\circledR}$, are affixed to the surface of each finger for. A picture of a sensor suite on a finger is shown in Fig. 5.

Each robot has the following nominal parameters: $l_{o}=$ $26.45 ", l_{1}=9.2 ", l_{2}=17.0 ", l_{3}=3.7 "$, and $l_{4}=17.05 "$, where the lengths are as shown in Fig. 3. In addition, the finger has length $l_{5}=6.0$ ". This length is the distance from the common intersection of axes 4,5 and 6 to the centroid

UNCLASSIFIED

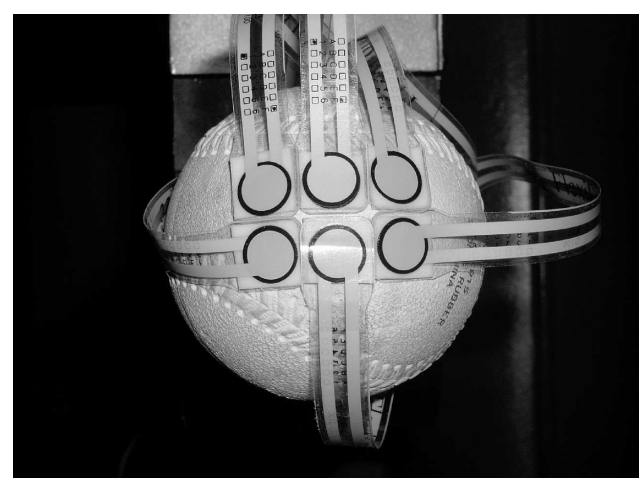

Fig. 5. Fingertip sensors.

of the ball that is the finger tip. The initial configuration for each robot with respect to a global palm frame is

$g_{p s_{1}}=\left[\begin{array}{cccc}0 & -1 & 0 & 47 \\ 1 & 0 & 0 & 14 \\ 0 & 0 & 1 & 0 \\ 0 & 0 & 0 & 1\end{array}\right] \quad g_{p s_{2}}=\left[\begin{array}{cccc}0 & 1 & 0 & 47 \\ -1 & 0 & 0 & 80 \\ 0 & 0 & 1 & 0 \\ 0 & 0 & 0 & 1\end{array}\right]$

where $g_{p s_{i}}, i=1,2,3,4$ represents the transformation from the palm to the station frame of robot $i$ and robots three and four are configured symmetrically with respect to robots one and two. The object's frame initially has the same orientation as the palm frame and is located at the center of the platform. Its height is dependent on the object. The entire layout is depicted in Fig. 6 where the station frames are represented with their relative configurations.

Here, the new vector fields, composed of Lie brackets, which replace the sliding velocities $v_{x}, v_{y}$, and the twisting velocity $\omega_{z}$ under rolling constraints are presented as well as the vector fields for a sphere rolling on a sphere for manipulation of a rubber ball. For the case of a sphere rolling on a sphere, the extended system is

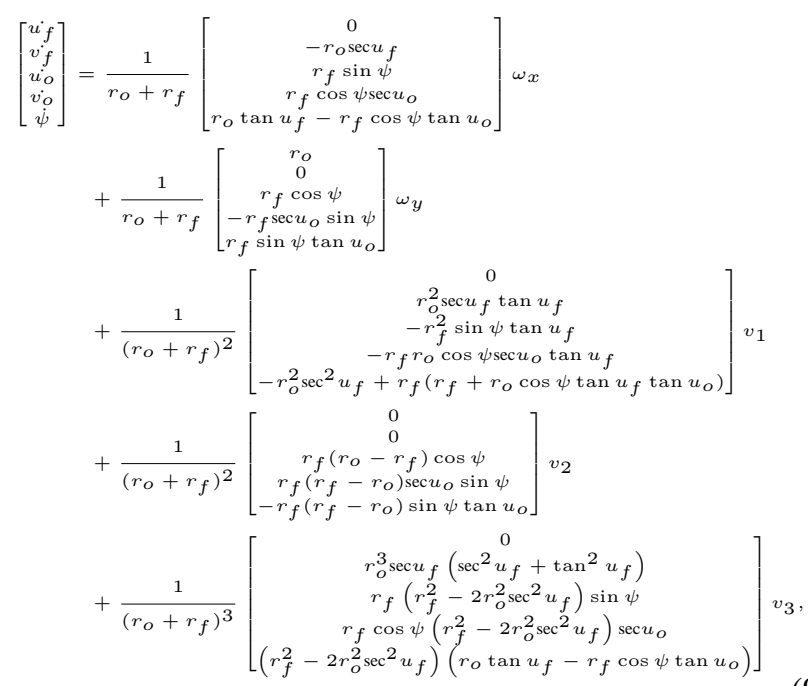

where $v_{1}, v_{2}$ and $v_{3}$ are the fictitious inputs that are approximated by piecewise constant inputs resulting in new displacements along their associated vector fields. 


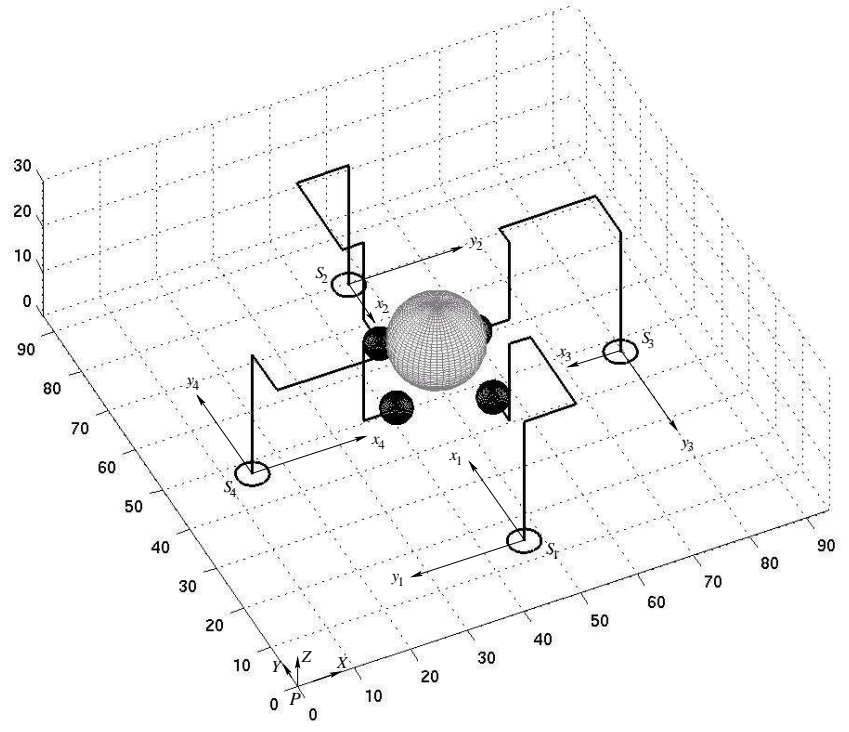

Fig. 6. Schematic of robotic manipulation test bed with reference frames.

The robots are controlled via a Pentium III, $500 \mathrm{MHz}$ computer running Linux Redhat release 7.2 containing three Galil 1880 motion control boards with 100-pin cable connectors. Each board has 8 analog input channels. The sensor readings are converted to a computer signal via a 16-bit analog-todigital converter with a range of $\pm 10 \mathrm{~V}$. Physically, the robots and sensors are connected to the boards through Galil ICM-1900 interconnect modules. Communication between the computer and the control boards is enabled by in-house device drivers [26].

The prototypical closed-loop manipulation process described in this paper is to acquire and lift the object, rotate the object, reposition the fingers based upon the open loop motion plan (including Lie bracket motions), and then to use the fuzzy inference system to adjust the grasp to account for modeling errors and external disturbances. The final three steps are repeated if the overall manipulation reconfiguration goal has not been met. Before the last three steps are repeated, however, the contact coordinate inputs to the motion planning algorithm are updated with current coordinates as measured by the sensors. Experimental data illustrating the trajectory the origin of the tool frame for one finger during manipulation step which illustrates this entire process is depicted in Fig. 7.

To acquire the object, the robots are commanded to a position outside the region of the object. Next, the fingers move based on output from the fuzzy controller to acquire the object. It is assumed contact occurs at a point and that this point is located at the geometric center of a sensor. If several sensors are in contact with the object, then the contact coordinate is taken as the centroid of the centers of the sensors measuring contact. Fig. 8 shows the locations of the sensors on a finger.

UNCLASSIFIED

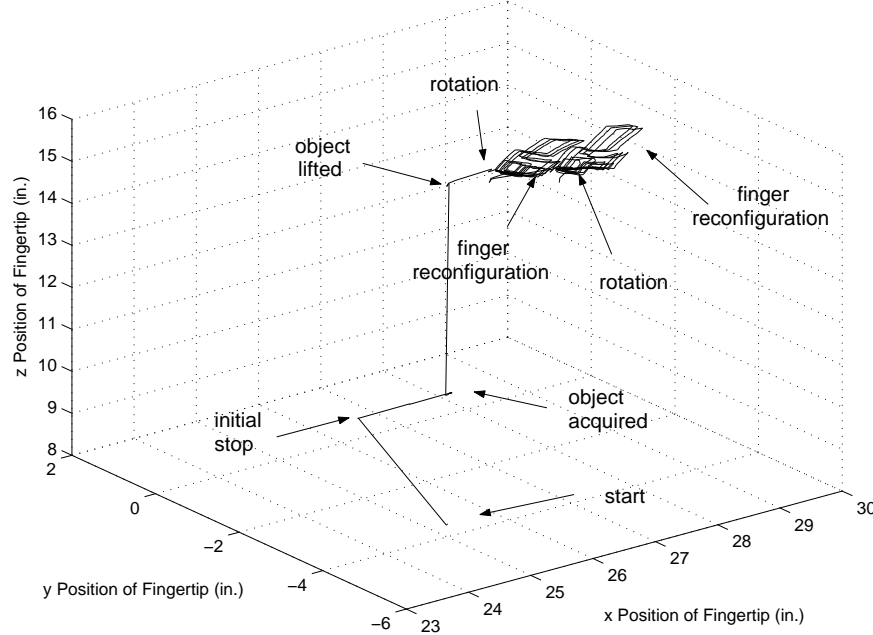

Fig. 7. General finger path during closed loop manipulation.

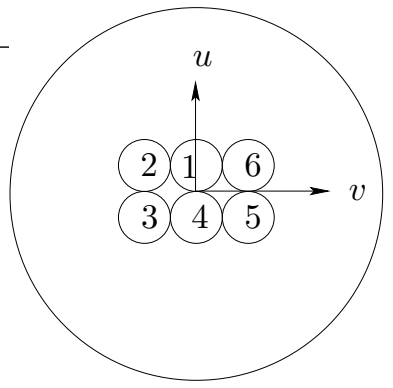

Fig. 8. Finger contact coordinates according to sensor locations.

The fuzzy controller contains two inputs, the current maximum contact force and the current $x$-position (in the station frame) of the fingertip, and one output, the change in the desired position of the fingertip. The membership functions for the input and output variables associated with manipulating the ball are shown in Fig. 9. This output is given by the first column of the fingertip's configuration with respect to the station frame $g_{s f_{i}}$. Therefore, the new desired position vector is

$$
p_{i}=\Delta\left[\begin{array}{lll}
R_{11} & R_{12} & R_{13}
\end{array}\right]^{T},
$$

where $\Delta$ is the fuzzy controller's output, and $R$ is the rotation matrix associated with the configuration $g_{s f}$. Closed form inverse kinematics are used to calculate the joint angles required to reposition the finger while maintaining a fixed orientation. This process is continued until $|\Delta|<0.05$ ". The rules are represented by the rule table shown in Fig. 10 and represent a simple balance between the maximum measured contact force and the position of the finger computed by the forward kinematics for the system. For example, if the finger is farther "in" than needed and the contact force is high, then it will move "out" a lot; however, if it is "in" more than expected but with a low contact force, then it will not move. In concert with the other four contact points, this would likely produce a more stable grasp, as indicated by the initial experimental results. It is anticipated that such 

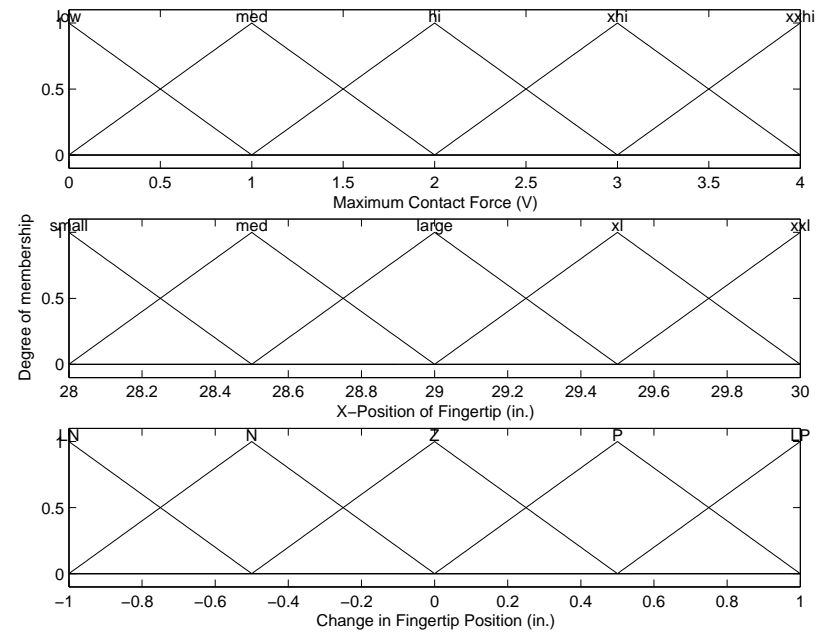

Fig. 9. Membership functions for fuzzy controller.

\begin{tabular}{|c|c|c|c|c|c|}
\hline \multicolumn{6}{|c|}{ Maximum Contact Force (V) } \\
\hline \begin{tabular}{|c|} 
Change \\
in \\
Position
\end{tabular} & low & med & hi & xhi & xxhi \\
\hline small & $\mathrm{LN}$ & $\mathrm{LN}$ & $\mathrm{LN}$ & NEG & ZERO \\
\hline med & $\mathrm{LN}$ & $\mathrm{LN}$ & NEG & ZERO & POS \\
\hline large & $\mathrm{LN}$ & NEG & ZERO & POS & LP \\
\hline $\mathrm{xl}$ & NEG & ZERO & POS & LP & LP \\
\hline $\mathrm{xxl}$ & ZERO & POS & LP & LP & LP \\
\hline
\end{tabular}

Fig. 10. Rule table for fuzzy inference system.

a logic structure would be of general applicability, but the focus of this paper is purely on manipulation by the described platform and more general applicability is not claimed.

Once the robots have grasped and lifted the ball, the configuration between each robot's station frame and the object is known since the fingertip and object share a common contact point. The configuration of the contact frame with respect to the object's frame for robot $i$ is

$$
g_{o l}=g_{s_{i} o}^{-1} g_{s_{i} f} g_{t f}^{-1} g_{t l} \text {. }
$$

The tool frame, $T$ is assumed to be at the fingertip, located on the surface of the finger along the extension of the $x$-axis of the $F$ frame, with the same orientation as $F$. The latter is determined from the forward kinematics using the robot's current joint angles.

Once the contact location is determined from Eq. 10, it must be rotated back by an amount equal to the current total rotation of the object to determine the correct contact coordinates since $g_{s_{i} o}$ is fixed in Eq. 10. The amount of

UNCLASSIFIED

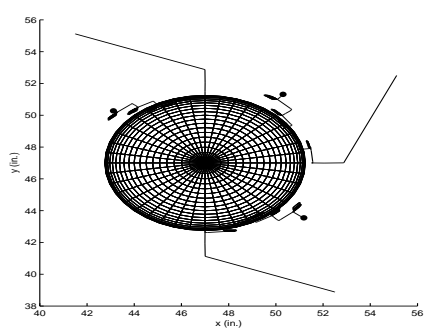

(a)

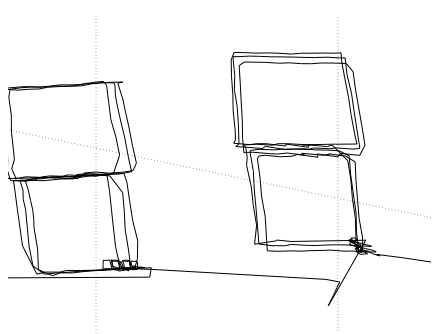

(b)
Fig. 11. Path followed by (a) fingers during manipulation experiment, and (b) exploded view of finger three's path in (a) showing correction by fuzzy controller, rotation, and Lie bracketing.

rotation is based on the desired value of the fixed-point rotation and assumption that the ball's configuration does not change during finger Lie bracketing. After each rotation the contact coordinates remain unchanged because the object rotates as well and the contact coordinates are determined relative to the object's frame. Since the finger has changed position relative to its station frame, however, the contact point must be calculated as if the ball remained fixed and the fingers repositioned. Then the point of contact must be rotated back so that the proper contact location is $p_{o l}=$ $R_{\omega}^{T}(\theta) \tilde{p}_{o l}$, where $R_{\omega}^{T}(\theta)$ is the rotation matrix about the general twist axis $\omega$ by an amount $\theta$ equal to the current total rotation of the object and $\tilde{p}_{o l}$ is the location of the contact point on the object as determined from Eq. 10.

The object's contact coordinates are then given by

$$
u=\operatorname{asin}\left(z_{o} / r_{o}\right) \quad \text { and } \quad v=\operatorname{atan} 2\left(y_{o}, x_{o}\right),
$$

where $x_{o}, y_{o}$, and $z_{o}$ are the $x-, y$-, and $z$-components of $p_{o l}$, respectively. Finally, the contact angle is

$$
\psi=\operatorname{atan} 2\left(-G_{o}^{x} \cdot G_{t}^{y}, G_{o}^{x} \cdot G_{t}^{x}\right),
$$

where $G_{o}^{x}$ and $G_{t}^{x}$ are the $x$-axes of the Gauss frames on the object and finger at the point of contact, respectively, and $G_{t}^{y}$ is the $y$-axis of the Gauss frame on the finger. This represents the local contact coordinates for an end-effector to roll or slide on the surface of the object.

\section{EXPERIMENTAL DEMONSTRATION}

To demonstrate the feasibility of the proposed method, some initial experiments involving both open and closed loop experiments were performed. For open loop experiments, the entire trajectory for each robot was computed offline. For the closed loop cases, the force sensors provided information to the fuzzy controller which adjusted the grasp after each set of Lie bracket motions. Experiments were conducted on several objects and the results in this paper are limited to those involving a spherical playground ball. Several open loop experiments were run to develop a baseline for comparing the closed loop results.

The trajectory of the robots during a manipulation is shown in Fig. 11. As a typical example, for a rotation about an axis through $(-1,1,1)$, the closed loop system was able to rotate the ball through $60^{\circ}$ while the open loop system 
was unable to achieve a rotation past $30^{\circ}$, where for five experiments for rotation about the axis $(-1,1,1)$, the average rotation for the open loop system was $17 \pm 8^{\circ}$, with a maximum of $30^{\circ}$.

It was typically the case that the closed-loop system demonstrated greater repeatability than the open-loop system, thus the control approach and experimental implementation seem to indicate the approach will be effective and robust. Current efforts are directed toward further experimentation and developing a simulation platform for the system, which will allow for thousands of simulations to be run which will provide the opportunity to collect a meaningful amount of statistical data regarding the efficacy of the presented method.

\section{CONCLUSIONS AND FUTURE WORK}

This work presented the theoretical framework for and the experimental demonstration of adding a fuzzy supervisory controller to a manipulation system. The main theoretical components that are put together to achieve this system are presented here as well as initial experimental results demonstrating that the system works and adding the fuzzy supervisory controller makes the system more robust resulting in more repeatable manipulation experiments. Current efforts are directed toward completely quantifying the extent of the improvement through systematic experimentation, including numerical simulation.

\section{REFERENCES}

[1] K. K. Choi, S. Li. Jiang, and Z. Li. Multifingered robotic hands: Contact experiments using tactile sensors. In Proceedings of the 1998 IEEE International Conference on Robotics and Automation, pages 2268-2273. IEEE, 1998.

[2] N. I. Glossas and N. A. Aspragathos. Fuzzy logic grasp control using tactile sensors. Mechatronics, 11(7):899-920, 2001.

[3] Bill Goodwine and Joel Burdick. Trajectory generation for kinematic legged robots. In IEEE International Conference on Robotics and Automation, pages 2689-2696, Albuquerque, NM, 1997.

[4] Bill Goodwine and Joel Burdick. Gait controllability for legged robots. In Proceedings of the 1998 International Conference on Robotics and Automation, pages 484-489, Leuven, Belgium, 1998. IEEE.

[5] Bill Goodwine and Joel Burdick. Controllability of kinematic systems on stratified configuration spaces. IEEE Transactions on Automatic Control, 46(3):358-368, 2001.

[6] Bill Goodwine and Joel Burdick. A general method for motion planning for quasi-static legged robotic locomotion and finger gaiting. IEEE Transactions on Robotics and Automation, 18(2):209-222, April 2002.

[7] L. Han, Y. S. Guan, Z. X. Li, Q. Shi, and J. C. Trinkle. Dextrous manipulation with rolling contacts. In Proceedings of the 1997 IEEE International Conference on Robotics and Automation, pages 992-997, Albuquerque, NM, 1997.

[8] Hiroaki Kobayashi. Control and geometrical considerations for an articulated robot hand. International Journal of Robotics Research, 4(1):3-12, 1985.

[9] Gerardo Lafferriere and Héctor J. Sussmann. A differential geometric approach to motion planning. In X. Li and J. F. Canny, editors, Nonholonomic Motion Planning, pages 235-270. Kluwer, 1993.

[10] Zexiang Li, Ping Hsu, and Shankar Sastry. Grasping and coordinated manipulation by a multifingered robot hand. International Journal of Robotics Research, 8(4):33-50, 1989.
[11] Daniel Liberzon and A. Stephen Morse. Basic problems in stability and design of switched systems. IEEE Control Systems Magazine, 19(5):59-70, October 1999.

[12] G.F. Liu, J. Li, and Z.X. Li. Coordinated manipulation of objects by multifingered robotic hand in contact space and active joint space. In Proceedings of the 2002 IEEE International Conference on Robotics and Automation, pages 3743-3748.

[13] G.F. Liu, J.J. Xu, and Z.X. Li. Automatic real-time grasping force determination for multifingered manipulation: Theory and experiments. In Proceedings of the IEEE/RSJ International Conference on Intelligent Robots and Systems, pages 1675-1680.

[14] Guanfeng Liu, Jijie Xu, , and Zexiang Li. On geometric algorithms for real-time grasping force optimization. 12(6):843-859, 2004.

[15] Daniel McNeill and Paul Freiberger. Fuzzy Logic. Simon \& Schuster, 1993.

[16] D. J. Montana. The kinematics of contact and grasp. The International Journal of Robotics Research, 7(3):17-32, 1988.

[17] R. M. Murray, Z. X. Li, and S. S. Sastry. A Mathematical Introduction to Robotic Manipulation. CRC Press, Boca Raton, Florida, 1994.

[18] Richard M. Murray and S. Shankar Sastry. Nonholonomic motion planning: Steering using sinusoids. IEEE Transactions on Automatic Control, 38(5):700-716, May 1993.

[19] Ciro Natale and Luigi Villani. Adaptive control of a robot manipulator in contact with a curved compliant surface. In Proceedings of the American Control Conference, pages 288-292, San Diego, California, June 1999.

[20] N. B. Petroff. Biomimetic Sensing for Robotic Manipulation. PhD thesis, University of Notre Dame, 2006.

[21] Mihir Sen and Bill Goodwine. Soft computing in control. In Mohamed Gad el Hak, editor, The MEMS Handbook, chapter 14. CRC Press, 2002.

[22] Mark W. Spong. Underactuated mechanical systems. In B. Siciliano and K. P. Valavanis, editors, Control Problems in Robotics and Automation, pages 135-150. Springer, 1998.

[23] Kazuo Tanaka, Masaki Iwasaki, and Hua O. Wang. Stable switching fuzzy control and its application to a hovercraft type vehicle. In Proceedings of the Ninth IEEE International Conference on Fuzzy Systems, volume 2, pages 804-809, San Antonio, Texas, 2000.

[24] J.C. Trinkle and R.P. Paul. Planning for dexterous manipulation with sliding contacts. The International Journal of Robotics Research, 9(3):24-48, 1990.

[25] J. Wang, S. J. Dodds, and W. N. Bailey. Co-ordinated control of multiple robotic manipulators handling a common object - theory and experiments. IEE Proceedings - Control Theory and Applications, 144(1):73-84, January 1997.

[26] Yejun Wei. Theoretical and Experimental Investigation of Stratified Robotic Finger Gaiting and Manipulation. PhD thesis, University of Notre Dame, 2002.

[27] Yejun Wei and Bill Goodwine. Stratified manipulation on nonsmooth domains. IEEE Transactions on Robotics and Automation, 20(1):128132, 2004.

[28] Alan M. Wing, Patrick Harggard, and Randall J. Flanagan. Hand and Brain: The Neurophysiology and Psychology of Hand Movements. Academic Press, Inc., 1996.

[29] Bin Yao and Masayoshi Tomizuka. Adaptive control of robot manipulators in constrained motion-controller design. Journal of Dynamic Systems, Measurement, and Control, 117(3):320-328, September 1995.

[30] Kuu-Young Young and Cheng-Chu Fan. Control of voluntary limb movements by using a fuzzy system. In Proceedings of the 32nd Conference on Decision and Control, pages 1759-1764, San Antonio, Texas, 1993. IEEE.

[31] L.A. Zadeh. Fuzzy sets. Inf. Contr., 8, 1965.

[32] L.A. Zadeh. Fuzzy algorithm. Inf. Contr., 12:94-102, 1968.

[33] L.A. Zadeh. Probability measures and fuzzy systems. J. Math. Anal. Appl., 23(2):421-427, 1968.

[34] Mohammad H. Zand, Payam Torab, and Arman Bahri. Hybrid position/force control of a dexterous hand based on fuzzy control strategy. In Proceedings of the 1997 IEEE 8th International Conference on Advanced Robotics, pages 133-139, Monterey, CA, July 7-9 1997. 